\title{
Platelet-rich plasma for patent ductus arteriosus: an orthopaedic surgeon's perspective
}

\author{
Mehmet Ali Engür, ${ }^{1}$ Defne Engür ${ }^{2}$ \\ ${ }^{1}$ Clinic of Orthopaedics, Isparta State Hospital; ${ }^{2}$ Neonatal Intensive Care Unit, Isparta Maternity and Children's \\ Hospital, Isparta, Turkey
}

\begin{abstract}
Although closure of ductus arteriosus requires multiple intriguing cell signalling pathways, current treatment options merely act through cyclooxygenase inhibition. Expectedly, medical management of ductus arteriosus is quite far away from optimum and there is an ongoing debate on its efficiency and safety. The ideal therapeutic agent for patent ductus arteriosus should target vascular remodelling and support the arrested molecular steps that should completely eventuate during anatomical closure.

Platelet-rich plasma injection, which has been used as a treatment alternative for many musculoskeletal conditions, can be a promising solution in this context by compensating the relative platelet dysfunction in preterms and providing a convenient microenvironment for proper cellular communication for ductal closure.

First, by inducing hyaluronan synthase, platelet-rich plasma can induce mount formation in the intima, which is the significant defective milestone in preterms. Second, by providing platelet-derived growth factor, it can further stimulate platelet agregation and occlusion of the lumen. Finally, it can provide an increment in local vascular endothelial growth factor and transforming growth factor- $\beta$ levels, which cannot be achieved because of insufficient intramural hypoxia. Migration of the vascular smooth muscles would further be triggered by transforming growth factor- $\beta$ and vascular endothelial growth factor, aiding a major contribution to ductal closure.

Above and beyond all other considerations, opportunity of local application would render maximum effectiveness and minimum side effects.
\end{abstract}

Keywords: Patent ductus arteriosus; platelet-rich plasma; preterm

Received: 24 September 2013; Accepted: 16 January 2014; First published online: 19 February 2014

$I^{2}$ NEALTHY TERM INFANTS, CLOSURE OF DUCTUS arteriosus occurs in two sequential steps. Initial constriction, mediated mainly by prostaglandin E2 withdrawal and oxygen exposure, is followed by platelet-triggered ductal sealing and subsequent vascular remodelling, which renders permanent closure. ${ }^{1}$ Although ductal constriction is achieved in preterm babies, remodelling and anatomical closure remains the cardinal problem leading to ductal reopening. Anatomical closure requires a

Correspondence to: D. Engür, Neonatal Intensive Care Unit, Isparta Maternity and Children's Hospital, Isparta, Turkey. Tel: +9024621171 11; Fax: +90246 21172 82; E-mail: defneka@hotmail.com ubiquitous cascade of cell signalling system, some of which are interrupted in preterms. Intramural hypoxia, production of various bioactive signalling agents, formation of intimal cushions, migration of smooth muscle cells, and platelet vessel wall interaction are the pivotal points for definitive ductal closure. $^{1,2}$

Current management of an open ductus relies upon cyclooxygenase inhibition, which provides vasoconstriction. However, it does not have any effect on vascular remodelling and permanent closure. Moreover, it has various systemic side effects on the developing systems of the premiee. ${ }^{2}$ Considering the common side effects and high failure rate of present 
medical agents, there is an urgent need for a novel therapeutic option targeting not only "vasoconstriction", but also "vascular reconstruction".

Platelet-rich plasma is a volume of plasma prepared by centrifugation of autologous blood and resulting in higher platelet levels than the peripheral blood concentration. Including higher concentration of platelets that provide an increased amount of several bioactive growth factors, platelet-rich plasma is reported to endorse wound healing, and thereby platelet-rich plasma injection has been used as a treatment alternative for many musculoskeletal conditions. ${ }^{3}$ The promising benefits of platelet-rich plasma for orthopaedic practice are because of promotion of the extracellular matrix formation, and collagen synthesis by various bioactive agents such as epidermal growth factor, vascular endothelial growth factor, insulin-like growth factor-1, plateletderived growth factor, fibroblast growth factor and transforming growth factor- $\beta$, and certain adhesive protein factors such as fibronectin. ${ }^{3}$ As the nature and fate of the cells can eventually be adjusted by modification of the extracellular matrix components with which they are in contact, platelet-rich plasma can warrant the ideal medium for proper signalling required to achieve anatomical closure.

\section{Four possible effects of platelet-rich plasma on ductus arteriosus}

1. Recently, Echtler et al ${ }^{4}$ provided a new view into the mechanisms of anatomical closure by demonstrating persistent ductus arteriosus in induced dysfunction of platelet adhesion and aggregation or defective platelet formation in mice. They have also reported mild thrombocytopaenia to be a significant risk factor for failure of ductus arteriosus closure in preterm infants. Studies illuminating the association between ductal patency and mean platelet volume and distribution width also supported this theory by highlighting the possible dysfunction of platelets as a contributing factor for ductal patency in extremely preterm infants. ${ }^{5}$ Platelet-rich plasma can be a promising solution in this context by utterly compensating the relative platelet dysfunction in preterms.

2. Inhibition of cyclooxygenase is the prevalent treatment for an open ductus. However, the complementary antenatal role of prostaglandin E2 has been disregarded. Prostaglandin E2 not only keeps it open until birth, but also prepares ductus for closure at birth by promoting intimal cushion formation. Therefore, cyclooxygenase inhibition in preterms, resulting in interruption of intimal cushion development, may conversely exacerbate the problem. ${ }^{2}$ Prostaglandin E2 controls migration of smooth muscle cells to form intimal mounts while stimulating synthesis and secretion of hyaluronic acid, an extracellular matrix component through which migration of smooth muscle cells occurs. ${ }^{2}$

The activity of hyaluronan synthetase enzyme in normal human mesothelial cells is regulated by certain molecules such as platelet-derived growth factor, transforming growth factor- $\beta$, epidermal growth factor, vascular endothelial growth factor, insulin-like growth factor-1, and fibroblast growth factor. It has been shown that exogenous platelet-derived growth factor resulted in stimulation of hyaluronan synthetase in mesothelial cells. ${ }^{6}$ Platelet-rich plasma, by inducing the local hyaluronan synthetase enzyme within the ductal wall, can support intimal mount formation.

3. Intramural hypoxia in the ductal wall, giving rise to cell death and production of particular cell signalling molecules including vascular endothelial growth factor, stimulates endothelial proliferation. It is the key trigger of remodelling; however, it is not achieved in preterms because of thin-walled ductus. ${ }^{1}$ Platelet-rich plasma can restore this relative deficieny of intrinsic bioactive agents and render a convenient microenvironment for proper cell signalling to perpetuate ductal closure.

4. Anatomical closure involves migration of the vascular smooth muscle cells into subendothelium, as well as increased deposition of collagen. Migration is dependent upon increased fibronectin levels and is further facilitated by chemotactic effects of transforming growth factor- $\beta$ and vascular endothelial growth factor. ${ }^{1,7}$ Platelet-rich plasma contains chemoattractants that can endorse migration.

\section{Proposed technique for platelet-rich plasma administration}

Preparation of platelet-rich plasma requires collection of autologous blood in adults; however, in a preterm newborn, it can readily be prepared by harvesting cord blood after the umbilical cord has been cut and is extracted from the foetal end of the cord. After preparation of the final product, local application can be possible by an umbilical arterial catheter. Umbilical arterial catheterisation is already a standard procedure for arterial access in extremely low birth weight infants, allowing an accurate monitorisation of the blood pressure, and provides an easy source for blood samples. It is usually placed at high position in the descending 
aorta, which corresponds to the thoracic vertebrae T6 and T9, which lies a few centimetres below the ductal opening. Advancing the catheter a few centimetres above the pre-determined depth for high position would allow us to locate its tip just in front of the aortic opening of ductus arteriosus; platelet-rich plasma infusion can then be performed.

Accessing the ductus from the aortic end is reasonable, as early ductal constriction occurs usually from the pulmonary end of the ductus and the direction of the shunt is mostly from the descending aorta to main pulmonary artery. The turbulent flow along the ductus would further help dispersion of bioactive agents along the lumen. Retrograde diastolic flow in the descending aorta in large ductal shunts would also help by diverting the blood along ductus. ${ }^{1}$

Finally, as safety is a critical concern in any neonatal study and as there are no reports about intravascular administration of platelet-rich plasma, the proposed method must be assessed in an animal model, which will also aid us to identify the minimum effective dose. One important aspect to remember while carrying out an experimental design regarding patent ductus arteriosus is to interpret the results carefully because of the physiological differences in the mechanism of ductus arteriosus closure between that species and humans. As closure of patent ductus arteriosus mostly depends upon vasoconstriction in small animals such as rodents, it seems to be wiser to choose a larger animal such as sheep or baboon in which ductal closure occurs with intimal mount formation. ${ }^{8}$

\section{Conclusion}

We propose that administration of platelet-rich plasma at the aortic end of the ductus arteriosus through an umbilical arterial catheter may help to restore the defective steps of ductal closure in preterm babies. Local application of platelet-rich plasma, instead of a systemic cyclooxygenase inhibitor, would provide maximum efficacy and minimum side effects.

\section{Acknowledgements}

None

\section{Financial Support}

This paper received no specific grant from any funding agency, commercial or not-for-profit sectors.

\section{Conflict of Interest}

None

\section{References}

1. Hamrick SE, Hansmann G. Patent ductus arteriosus of the preterm infant. Pediatrics 2010; 125: 1020-1030.

2. Ivey KN, Srivastava D. The paradoxical patent ductus arteriosus. J Clin Invest 2006; 116: 2863-2865.

3. Lee KS, Wilson JJ, Rabago DP, Baer GS, Jacobson JA, Borrero CG. Musculoskeletal applications of platelet-rich plasma: fad or future? Am J Roentgenol 2011; 196: 628-636.

4. Echtler K, Stark K, Lorenz M, et al. Platelets contribute to postnatal occlusion of the ductus arteriosus. Nat Med 2010; 16: 75-82.

5. Alyamac Dizdar E, Ozdemir R, Nur Sari F, et al. Low platelet count is associated with ductus arteriosus patency in preterm newborns. Early Hum Dev 2012; 88: 813-816.

6. Heldin P, Asplund T, Ytterberg D, Thelin S, Laurent TC. Characterization of the molecular mechanism involved in the activation of hyaluronan synthetase by platelet-derived growth factor in human mesothelial cells. Biochem J 1992; 283: 165-170.

7. Koppel R, Rabinovitch M. Regulation of fetal lamb ductus arteriosus smooth muscle cell migration by indomethacin and dexamethasone. Pediatr Res 1993; 33: 352-358.

8. Coceani F, Baragatti B. Mechanisms for ductus arteriosus closure. Semin Perinatol 2012; 36: 92-97. 